\title{
A Successful Multidisciplinary Intensive Care Approach of Anaerobic Septic Shock caused by Descending Necrotising Mediastinitis

\author{
Dijana Popevski ${ }^{1}$, Petar Ugurov ${ }^{2}$, Rodney Rosalia', Dragica Vuckova', Dashurie Neziri², Emil Stoicovski', Zan Mitrev ${ }^{4}$
}

${ }^{\prime}$ Intensive Care Unit, ${ }^{2}$ Department of Cardiology, ${ }^{3}$ Department of semi Intensive Care Unit, ${ }^{4}$ Department of Cardiovascular Surgery, Zan Mitrev Clinic, Skopje, Republic of Macedonia

\section{Background}

- Descending Necrotising Mediastinitis (DNM) is an acute, lifethreatening disease caused by infections originating in the head and neck area. Antibiotherapy and surgical debridement of the affected tissue are the cornerstones of treatment.

\section{Case Presentation}

- 38-year old male

- Lacunar angina

- Abscesses in the anterior and middle mediastinum region and the distal part of the neck, directly on the border with the left lobe of the thyroid gland, (Fig. 1).

- Hemocultures confirmed Streptococcus alfa haemolyticus and Clostridium species positive cultures. PCT $20.5 \mu \mathrm{g} / \mathrm{L}, \mathrm{CRP}$ $325.3 \mathrm{mg} / \mathrm{L}, \mathrm{WBC} 19.8^{*} 103$ cells $/ \mu \mathrm{L}$, Lactate $6,3 \mathrm{mmol} / \mathrm{L}$, SVR $<500$ dynes/sec/cm 5 and the SAPS II score of 63 pointed to a case of severe sepsis with a poor prognosis.

\section{Primary Treatment}

- Aggressive antibiotic regimen (Fig. 2), transthoracic and mediastinal surgical evacuation of the abscess, vacuum sealing drainage with a pleural chest tube.

\section{Adjuvant Treatment}

- Continuous Venovenous Hemofiltration with CytokineAdsorbing Filters (CVVHF-CAH) set at a flow rate of $35 \mathrm{~mL} /$ $\mathrm{kg} / \mathrm{h}$ for 48 hours.

- Extracorporeal blood oxygenation and ozonation (EBOO) as an alternative (adjuvant) therapy for one hour through the right femoral vein.
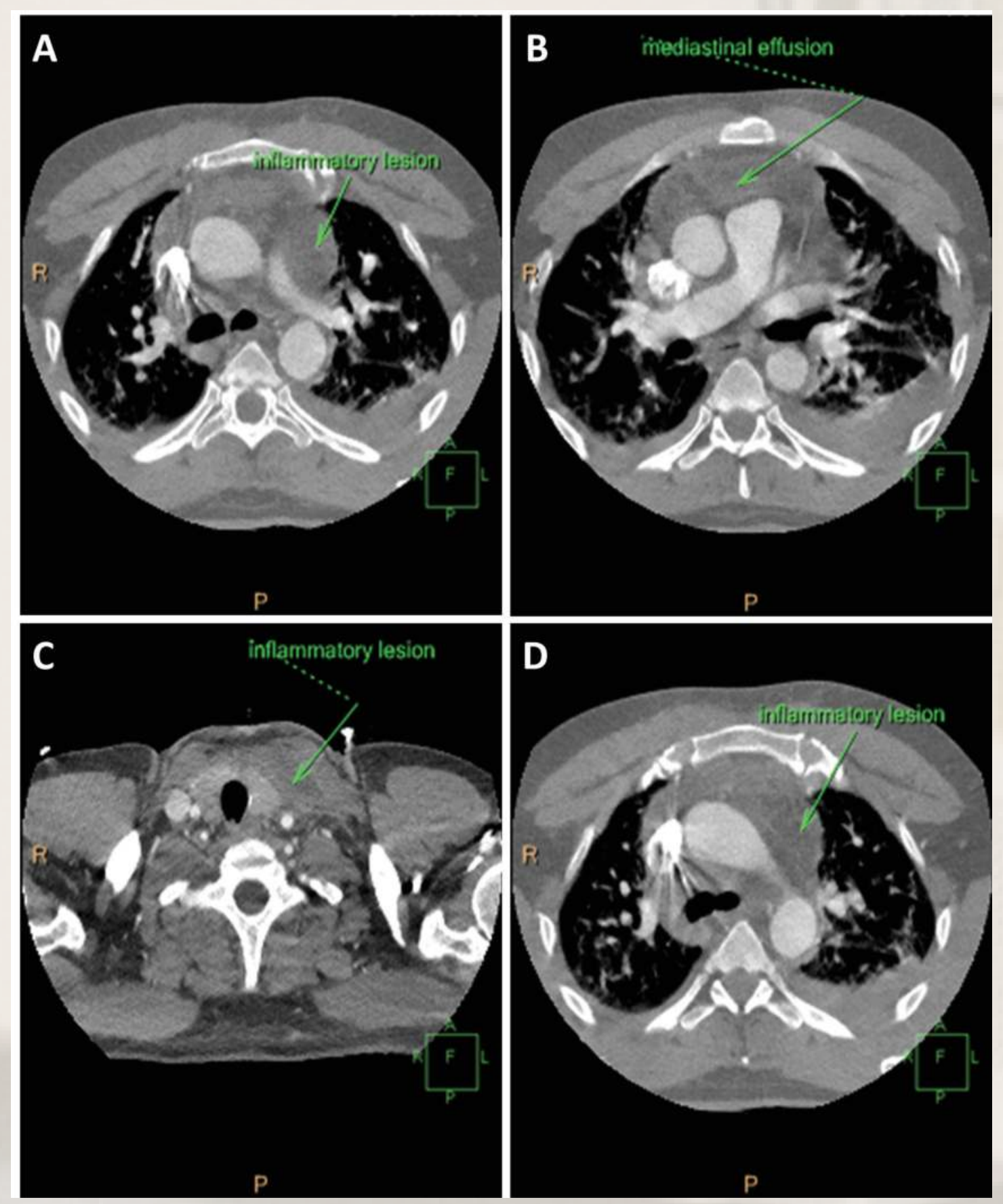

Figure 1. Pre-operative CT scans of a critically ill patient displaying clinical signs of descending necrotising mediastinitis. A and B) increased density of the middle mediastinal space. Fluid collections in mediastinum and lymphadenopathy with B) bilateral pleural effusions. C and D) fluid collections, abscess formation left of the thyroid gland and ventral of the carotid arteries (arrow).

\section{Clinical Outcome}

- Total hospitalisation was 39 days; combination therapy steadily improved the patient's overall condition and reduced sepsis biomarkers (Fig. 2). Follow up at dat 44 (Fig. 3) showed complete resolution of abscesses.

- 1 year follow confirmed a full recover in the absence of complications.

\section{Discussion}

DNM is a rapidly progressing life-threatening disease. Mediastinal infection can induce lethal septicaemia if not diagnosed and managed appropriately. Each case requires a personalised treatment modality.

\section{Learning points}

Effective management of DNM-induced anaerobic septic shock requires a multidisciplinary therapy based on the timely administration of antibiotics, skilled surgical intervention and the use of adjuvant treatment modalities to support renal, pulmonary and hemodynamic functioning.
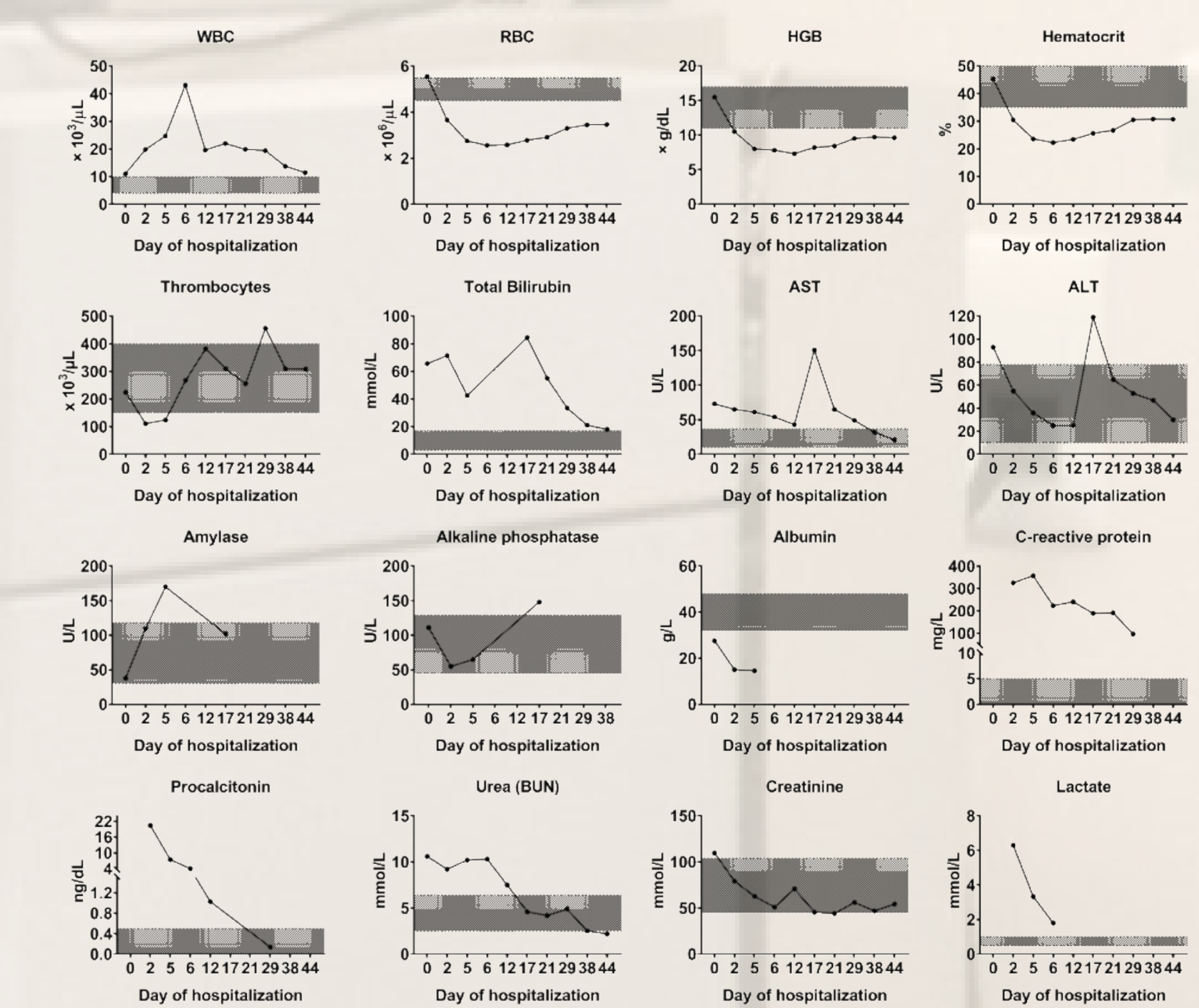

Day of hospitalization

Figure 2. Kinetics of Laboratory parameters Changes in biochemical parameters associated with Heart Failure and multi organ failure over time. Hospital discharge was on day 39. Follow up controls were performed on day 44. Grey shaded area represents normal reference ranges.
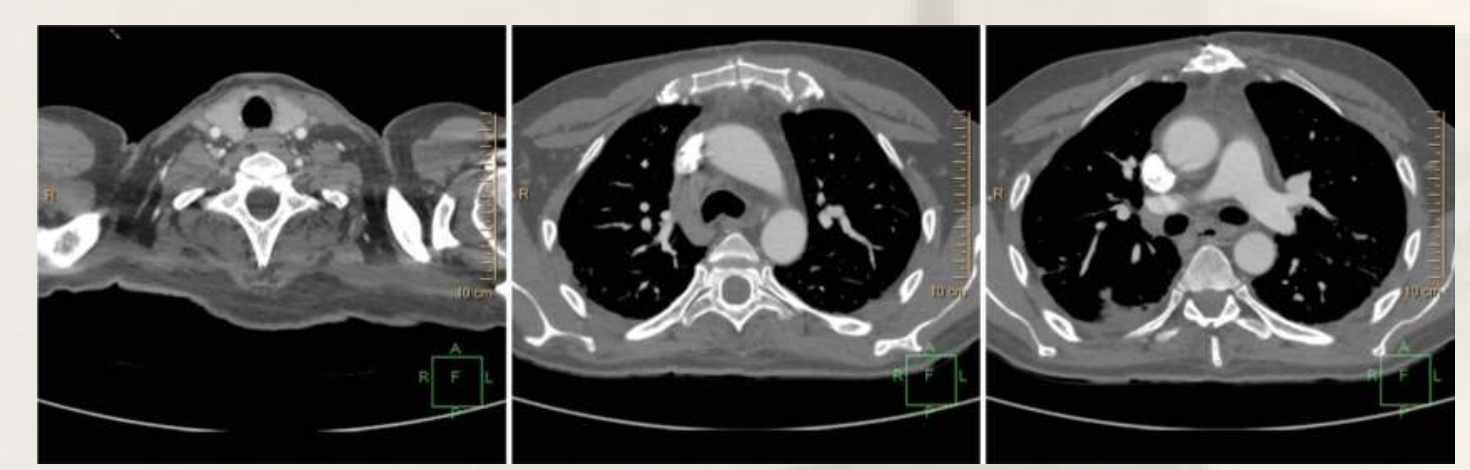

Figure 3. Control CT analysis A) Control CT scan performed on day 44 shows normal physiological findings indicating a complete regression of the abscess formation near the left lobe of the thyroid gland and ventral of the carotid arteries. Panels displayed in B) and C) show fibrous adhesion in the middle mediastinal space. 